\section{Balanced regulation of synthetic biology}

Contrary to the opinion of Genya Dana and colleagues (Nature 483, 29; 2012), the greater sophistication of modified microbes created by synthetic biology could make them less, not more, difficult to regulate, manage and monitor than their naturally occurring counterparts.

Several routes could lead to a hypothetical disaster scenario, in which artificially produced microorganisms spread unchecked in the environment (see go.nature.com/pncgxs). But we need to take into account that some of these paths would be favoured over others, and that ecological and other interactions might attenuate or amplify those different probabilities. Synthetic biology could then be used to engineer environmental safety into these organisms.

There is a danger that adverse public opinion could hinder the development of synthetic biology's potential. To prevent this, the media must refrain from hyping the benefits or risks of the technology. That will ensure that synthetic biology can be developed safely under a regulatory system that is based on the probability of occurrence of hazardous events, rather than on imagined possibilities.

The questions raised by Dana et al. should be considered as part of any risk-governance system for synthetic biology. Governance must be adaptive and scientifically and economically robust, given that most outcomes of technological innovation are still speculative. At the Economic and Social Research Council's Innogen Centre in Edinburgh, UK, we are developing such a strategy, to enable beneficial innovation with minimal risk to people and the environment while balancing stakeholder interests (see go.nature.com/khvykc).

Dana et al. propose that an investment of US\$20-30 million over 10 years is needed to assess the risks associated with synthetic microbes. But, in our view, this is not yet justified. A more balanced and wideranging analysis is called for before major funding decisions are made relating to governance of the technology.

Joyce Tait, David Castle ESRC Innogen Centre, University of Edinburgh, UK.

joyce.tait@ed.ac.uk

\section{Media centre: vital to US science}

In my view, Colin Macilwain's reservations over the challenges facing the planned US Science Media Center are overly pessimistic (Nature 483, 247; 2012).

No one - least of all the members of an exploratory committee for such a centre in the United States (including myself) - would deny that real differences exist between Britain and the United States in our cultures, politics, science and media. If established, a US centre (see www. sciencemediacenter.org) would embrace a uniquely American model of operation to serve the country's journalists and public understanding of science. It would adapt to its cultural landscape, just as those of Canada or Japan have.

It is precisely because of the bitter contention in the United States over issues such as climate change, stem-cell research and evolution that we need a science media centre now more than ever.

Successful science media centres in other countries have demonstrated that they improve civic discourse, because journalists are better informed about the science behind the controversies. Policy-makers can make decisions based on the best available science, and citizens can make smarter personal life choices and engage in serious political dialogue.

Julia A. Moore Woodrow Wilson International Center for Scholars, Washington DC, USA.

mooreblaney@gmail.com

\section{Media centre: more than public relations}

As chief executive of London's

Science Media Centre, I find the prospect of a similar centre in the United States more exciting than Colin Macilwain seems to (Nature 483, 247; 2012). A network of science media centres (SMCs) is already flourishing in Japan, Canada, Australia, New Zealand and the United Kingdom, with Denmark soon to join the list. This model works and, given the calibre of the people involved in the US move, I am confident that they will be able to meet any challenges head-on.

The SMC model is popular with journalists because it bucks public-relations protocol. None of us promotes our brand name or any institutional message, we are just as happy to kill stories as to create them, and we embrace difficult and controversial issues. This special mix is likely to be as popular with US journalists as it is with those in the other countries.

Science journalists use quotes from SMCs and/or treat them as a source of background information. If US science journalists are less likely to use quotes, as Macilwain implies, then a US SMC would simply operate differently. However, a scarcity of specialist science journalists may force a US SMC to operate more like the Australian centre, which tends to work with general news reporters.

SMCs around the world will have to adapt to different cultures, in which attitudes to acceptable funding sources may vary. But what really matters is that SMCs should be independent of those funders, whether they be from industry, government, the media or the scientific community. Fiona Fox Science Media Centre, London, UK.

fiona@sciencemediacentre.org

\section{Journals' role in ethical research}

False evidence in medical research can endanger lives, so countermeasures must be stepped up - particularly in light of recent cases (see go.nature.com/9ivjnm).

Improving scrutiny and verifying source data would be prohibitively costly and time-consuming, even if it were legally feasible. Instead, journals should demand proof before publication that research has been approved by a recognized body, such as an independent ethics committee or institutional review board, so that fraudulent claims can be identified (see, for example, Nature 477, 384; 2011). This approval is legally required in most countries and has now become an international standard (see go.nature.com/ krrr6g), so is easy to obtain.

Authors and their institutions should inform the journal promptly should their results be later invalidated, and permit inspection or auditing of reports if necessary (S. Kleinert and R. Horton Lancet 372, 789-790; 2008). This would speed publication of the formal retraction to correct the scientific record.

Holger Baumgartner Research Ethics Committee, Innsbruck Medical University, Austria. holger.baumgartner@i-med.ac.at Competing financial interests declared (see go.nature. com/5qlre5).

CONTRIBUTIONS

Correspondence may be sent to correspondence@ nature.com after consulting the guidelines at http:// go.nature.com/cmchno. 